\title{
BIIDXI, the At4g32460 DUF642 gene, is involved in pectin methyl esterase regulation during Arabidopsis thaliana seed germination and plant development
}

\author{
Esther Zúñiga-Sánchez', Diana Soriano', Eleazar Martínez-Barajas², Alma Orozco-Segovia' \\ and Alicia Gamboa-deBuen ${ }^{1 *}$
}

\begin{abstract}
Background: DUF642 proteins constitute a highly conserved family of proteins that are associated with the cell wall and are specific to spermatophytes. Transcriptome studies have suggested that members of this family are involved in seed development and germination processes. Previous in vitro studies have revealed that At4g32460- and At5911420-encoded proteins interact with the catalytic domain of pectin methyl esterase 3 (AtPME3, which is encoded by At3g14310). PMEs play an important role in plant development, including seed germination. The aim of this study was to evaluate the function of the DUF642 gene At4g32460 during seed germination and plant development and to determine its relation to PME activity regulation.
\end{abstract}

Results: Our results indicated that the DUF642 proteins encoded by At4g32460 and At5g11420 could be positive regulators of PME activity during several developmental processes. Transgenic lines overexpressing these proteins showed increased PME activity during seed germination, and improved seed germination performance. In plants expressing At4g32460 antisense RNA, PME activity was decreased in the leaves, and the siliques were very short and contained no seeds. This phenotype was also present in the SALK_142260 and SALK_054867 lines for At4g32460.

Conclusions: Our results suggested that the DUF642 family contributes to the complexity of the methylesterification process by participating in the fine regulation of pectin status during plant development.

Keywords: DUF642 proteins, Pectin methyl esterases, Germination

\section{Background}

DUF642 proteins constitute a highly conserved family of cell wall-associated proteins specific to spermatophytes [1]. Although proteins in this family have been detected in cell-wall proteomes from a variety of plants and tissues, only one functional study on this protein family has been published so far. At5g25460 is highly expressed in seedlings during the early developmental stages, and plants of the At5g25460-null mutant have shorter roots and smaller rosettes than those of wild-type plants [2].

\footnotetext{
* Correspondence: agamboa@ecologia.unam.mx

'Instituto de Ecología, Universidad Nacional Autónoma de México, Apartado Postal 70-275, Ciudad Universitaria, México 04510, Distrito Federal Mexico Full list of author information is available at the end of the article
}

Transcriptome analyses have revealed the differential expression of five DUF642 genes during seed barley germination, suggesting a possible function of this protein family during the germination process [3]. In addition, differential spatial expression of DUF642 genes among various seed compartments during germination has been reported in Arabidopsis thaliana. A gene expression study showed that At3g08030 and At5g11420 transcripts are enriched in the micropylar endosperm before testa rupture, whereas At4g32460 is expressed in this compartment after testa rupture [4]. In Brassica oleracea seeds, the expression of the At5g25460 gene ortholog increases during germination [5]. At3g08030 transcript is present in after-ripened seeds, and the transcript levels increased in seeds subjected to controlled imbibitions in 
soil or water (matrix-primed and hydroprimed seeds). Notably, At3g08030 transcript is absent from aged seeds with low germination performance [6].

DUF642 proteins have been detected in the cell-wall proteomes of multiple tissues [7]. A transcriptome analysis of stigmatic papillae cells revealed high transcript levels of two DUF642 genes, At4g32460 and At2g41800, and genes encoding other cell wall-related proteins, including a pectin methyl esterase. Cell wall remodeling of the stigma is involved in successful pollination, likely via regulating the penetration of the pollen tube through the transmitting tract [8]. In Lilium longiflorum, an analysis of proteins in the stigmatic exudate revealed a DUF642 protein [9].

Proteins encoded by At4g32460 and At5g11420 interact in vitro with the catalytic domain of pectin methyl esterase 3 (AtPME3, encoded by At3g14310) [10]. Protein interactome data have proved to be an useful resource for formulating and testing hypotheses [11]. One potential physiological function of the DUF642 proteins encoded by At4g32460 and At5g11420 is related to the regulation of PME activity. Several studies have shown that the degree of pectin methylesterification, a highly regulated process, is critical for fine-tuning the biomechanical properties of the cell wall during various developmental processes [12-14]. The demethylesterification of pectins is mediated by PMEs, and PME catalytic activity is regulated by PME inhibitor (PMEI) proteins [15].

Unesterified pectins, especially homogalacturonans (HGs), are the substrates for polygalacturonases (PGs), enzymes regulated by polygalacturonase inhibitor proteins (PGIPs) that are involved in cell separation processes [16]. Differences in pectin methylesterification have been described during pistil, silique, and seed development. In olive, low methylesterified HGs are detected in the stigma and in the transmitting tissue during pollination [17]. In A. thaliana, silique growth is related to a decrease in the degree of methylesterification [18]. In seeds of A. thaliana, the cell walls within the embryo have low levels of unesterified pectins, the endosperm cell walls contain abundant unesterified HG, and the testa cell walls are rich in highly methylesterified HG [19]. In A. thaliana, the genes encoding pectin-modifying enzymes and their regulators are highly regulated during the first $24 \mathrm{~h}$ of seed germination [20]. In yellow cedar seeds, PME activity positively correlates with germination performance [21].

In $A$. thaliana and related endospermic species, germination is a two-step process that requires testa and endosperm rupture for radicle protrusion [22]. During $A$. thaliana germination, PME activity increases until testa rupture is complete, and decreases during endosperm breakdown. Overexpression of PMEI led to accelerate endosperm breakdown and an improved capacity for radicle emergence. Delays in endosperm rupture caused by abscisic acid significantly extend the period of high PME activity [13]. Conversely, PGIP overexpression inhibits germination, a process that is enhanced in pgip mutant seeds [23].

The aim of this study was to study the function of the DUF642 gene At4g32460 during seed germination and plant development. We evaluated the role of the BDX protein in the regulation of PME activity, focusing on the periods of seed germination and plant growth. We demonstrated that the overexpression of either At4g32460 or its homolog At5g11420 increased PME activity and promoted germination, primarily by accelerating testa rupture. We also demonstrated that total PME activity was inhibited in At4g32460 antisense transgenic plants and that the morphological changes in these plants included small siliques with no seeds. This phenotype was also observed in SALK T-DNA mutants. In accordance with these results, we named At4g32460 as BIIDXI (BDX), which means 'seed' in the Zapotec language. Our data suggest that DUF642 proteins are involved in the regulation of PME, thereby remodeling the cell wall during various processes in plant development.

\section{Results}

BIIDXI is expressed in the embryos of imbibed seeds, roots, leaves, stems, and various floral organs (bar.utoronto.ca). To determine whether the cloned region (Additional file 1: Figure $\mathrm{S} 1 \mathrm{C}$ ) was sufficient to drive expression in a pattern similar to that described previously for A. thaliana, we produced transgenic plant lines containing the cloned fragment fused to the green fluorescent protein (GFP) reporter. Three transgenic lines were produced, and we monitored GFP fluorescence throughout their growth and development. GFP driven by the $B D X$ promoter was highly expressed in the vascular tissue of primary and lateral roots, and in leaves, stamens, and petals (Figure 1). GFP fluorescence was detected in the vascular tissue of radicles from seeds that had been germinating for $48 \mathrm{~h}$ and $72 \mathrm{~h}$ (Figure 1A and B). During the seed imbibition process, GFP fluorescence was detected from $6 \mathrm{~h}$ until germination was complete (Additional file 1: Figure S2). In the primary roots of 6-day-old seedlings, GFP fluorescence was detected exclusively in the provascular tissue of the meristematic and transition zone (Figure 1C). In the roots of 8-day-old seedlings, GFP fluorescence was detected in pericycle cells in the differentiation zone (Figure 1D). In the roots of 22-day-old plants, GFP fluorescence was detected in the vascular tissue in specific regions of the mature zone (Figure 1E). GFP fluorescence was also detected in the vascular tissue of fully expanded leaves (Figure 1F). During different stages of flower development, $B D X$ promoter-driven GFP expression was detected exclusively in the vascular tissue of stamen filaments and anthers, in petals, and in the stigmatic papilla, as described previously [8] (Figure 1G and $\mathrm{H}$ ). 


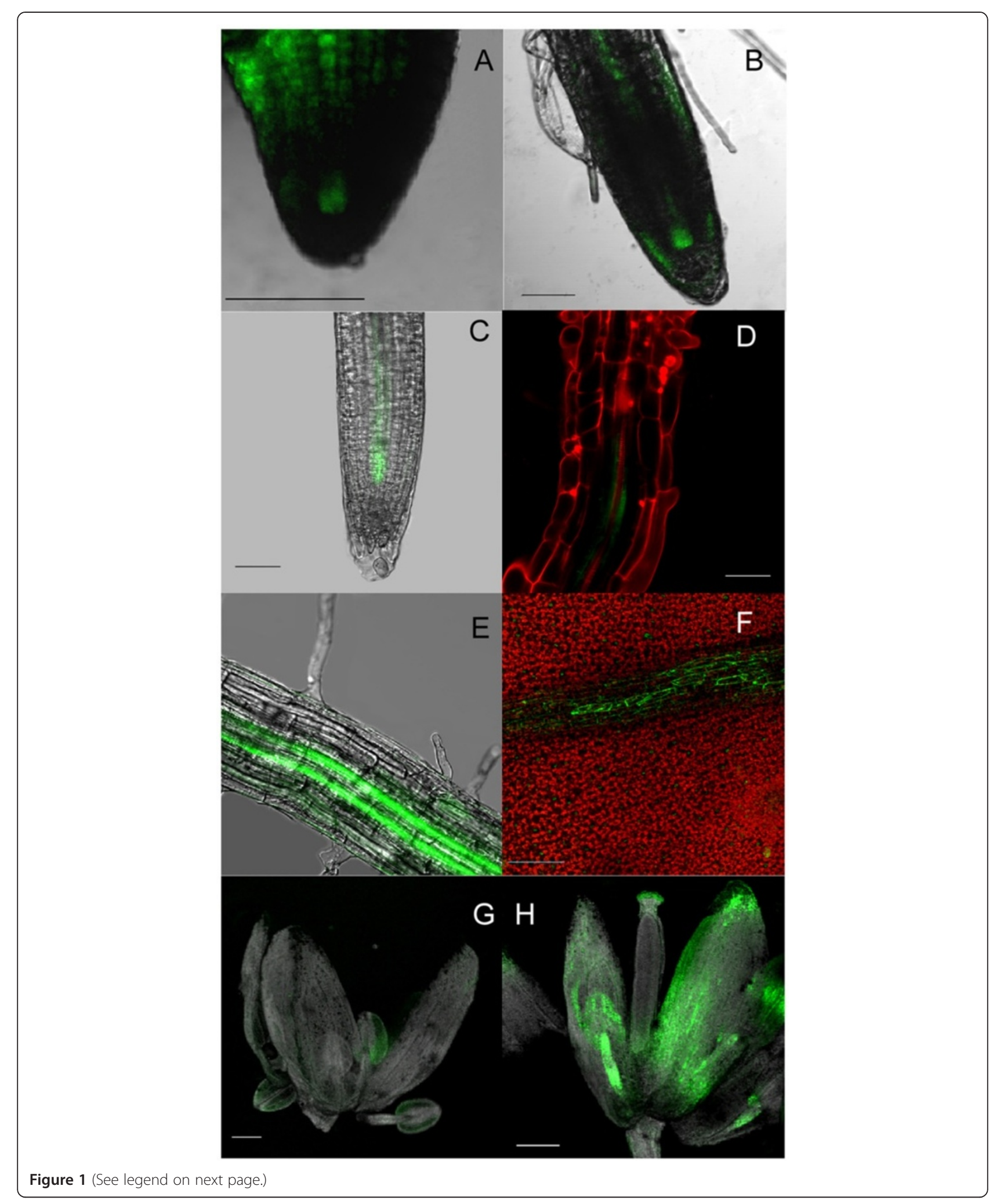


(See figure on previous page.)

Figure 1 Identification of At4g32460 promoter activity during Arabidopsis thaliana development using pBDX::ER-GFP plants

(Additional file 1: Figure S1C). A) GFP fluorescence in radicle tissue of a 48-h germinating seed. B) GFP fluorescence in radicle tissue of a 72-h germinating seed. C) GFP fluorescence in different cell types of meristematic and transition zones of primary roots from 4-day-old seedlings. D) GFP fluorescence in vascular tissue of maturation zone of primary roots of 22-day-old plants. E) GFP fluorescence in pericycle-differentiated cells that constitute primordium of lateral root emerging from maturation zone of primary roots of 22-day-old plants. F) GFP fluorescence in vascular tissue of fully expanded leaves. G) GFP fluorescence in anthers and petals of stage-6 flowers. H) GFP fluorescence in vascular tissue of stamen filaments, anthers, petals, and stigma of stage- 12 flowers. Scale bars $=50 \mu \mathrm{m}$ in A and B, $15 \mu \mathrm{m}$ in $\mathrm{D}, 100 \mu \mathrm{m}$ in $\mathrm{E}$ and $\mathrm{F}$, and $300 \mu \mathrm{m}$ in C, G, H, and I. Images A, B, E, F, G, and $\mathbf{H}$ are projections of confocal Z-stacks. C and D are longitudinal sections.

Next, we analyzed $B D X$ promoter activity in embryos at various stages; heart stage, torpedo stage, and mature embryos (Figure 2). GFP fluorescence was detected in provascular cells from the radicle meristematic region of the mature embryo (Figure 2A) and in embryos at the torpedo (Figure $2 \mathrm{C}$ ) and heart stages (Figure 2E). The expression pattern of GFP driven by the $B D X$ promoter was primarily associated with vascular tissue during different stages of plant development, consistent with previous reports.

Transcriptome analyses have revealed that $B D X$ expression is induced by auxin [24] and also by gibberellic acid (GA) during germination [25]. In silico analysis of the putative promoter region ( $\mathrm{pBDX}$ ) revealed at least two auxin response factor motifs [26] and two gibberellic acid response element (GARE) [27]. We performed hormone induction analyses to test whether the pBDX fragment contained information for auxin and GA responses. Auxin and GA treatments, for $2 \mathrm{~h}$ or $48 \mathrm{~h}$, altered $B D X$ expression in the roots of 7-day-old seedlings. In both treatments, $B D X$ expression was detected in vascular tissue, as previously observed in control seedlings, but also in cortical cells (Additional file 1: Figure S3).

To understand the physiological function of $B D X$, we generated overexpression lines (OEBDX; Figure 3) in which the full-length At4g32460 coding sequence was expressed under the control of the cauliflower mosaic virus $35 \mathrm{~S}$ promoter (Additional file 1: Figure S1A). Several independent and homozygous transgenic lines were obtained. We examined dry seeds from two lines to determine their PME activity and $B D X$ transcript levels.

$B D X$ transcript was not detected in wt dry seeds, but was presented at high levels in the overexpression lines (Figure 3A). Although there were no morphological differences among the different lines at all developmental stages (results not shown), there was increased total PME activity in vegetative-meristem-enriched samples and in seedlings $(p<0.001)$, compared with that in wt. There was no significant difference in PME activity in leaves between the overexpression lines and wt (Figure 3B). In wt $A$. thaliana seeds, PME activity has been reported to increase before testa rupture and to decrease at the beginning of endosperm rupture [13]. Based on this information, we performed a germination analysis of matrix-primed seeds of two OEBDX lines and wt plants. Plants transformed with the empty vector were used as a negative control (Additional file 1: Figure S4). Compared with wt, OEBDX lines showed a shorter initial time of testa rupture $(p=0.02$, Figure $3 \mathrm{C})$, and their seeds showed a shorter time to endosperm break initial time ( $p=0.004$, Figure 3D).

Phylogenetic studies have demonstrated that $B D X$ and At5g11420 resulted from a recent duplication event, and that their respective encoded proteins interact with AtPME3 in vitro $[1,10]$. Also, recent studies have shown that At5g11420 expression in the micropylar endosperm increases prior to testa rupture, suggesting a potential role for At5g11420 in this process [4]. To evaluate the possible role of $A t 5 g 11420$ in testa rupture, we generated overexpression lines (OE11420, Figures 1B and 4). There were no morphological differences among the different lines and wt (results not shown). At5g11420 transcript was detected at high levels in dry OE11420 seeds (Figure 4A). Compared with wt, the OE11420 lines showed a significant increase in PME activity in vegetative-meristem-enriched samples and seedlings $(p=0.003)$, but no significant change in PME activity in the leaves (Figure 4B). Also, OE11420 matrix-primed seeds showed improved germination, compared with that of wt seeds (Figure 4C and D). Specifically, the lag time until the initiation of testa and endosperm rupture was shorter in OE11420 seeds than in wt seeds ( $p=0.004$ and $p<0.001$, respectively). There was an increase in the rate of endosperm rupture in OE11420 lines $(p<0.001)$. Germination analysis of OEBDX and OE11420 matrix-primed seeds suggests that the overexpression of these genes in dry seeds improves their germination, possibly through enhancing testa rupture performance (Figures 3 and 4).

To determine the effects of the overexpressed genes on seed germination and PME activity during germination, we tested seeds without a priming pre-treatment. The initial testa rupture and endosperm rupture times were shorter for OE seeds than for wt seeds $(p=0.01$ and $p<0.01$, respectively). The seeds of OEBDX and OE11420 did not show different testa rupture rates, but seeds of both lines showed significantly lower endosperm rupture rates compared with that of wt seeds ( $p=0.01$, Figure $5 \mathrm{~A}$ and B). For wt seeds, PME activity increased before testa rupture was complete and decreased thereafter, similar to the pattern 


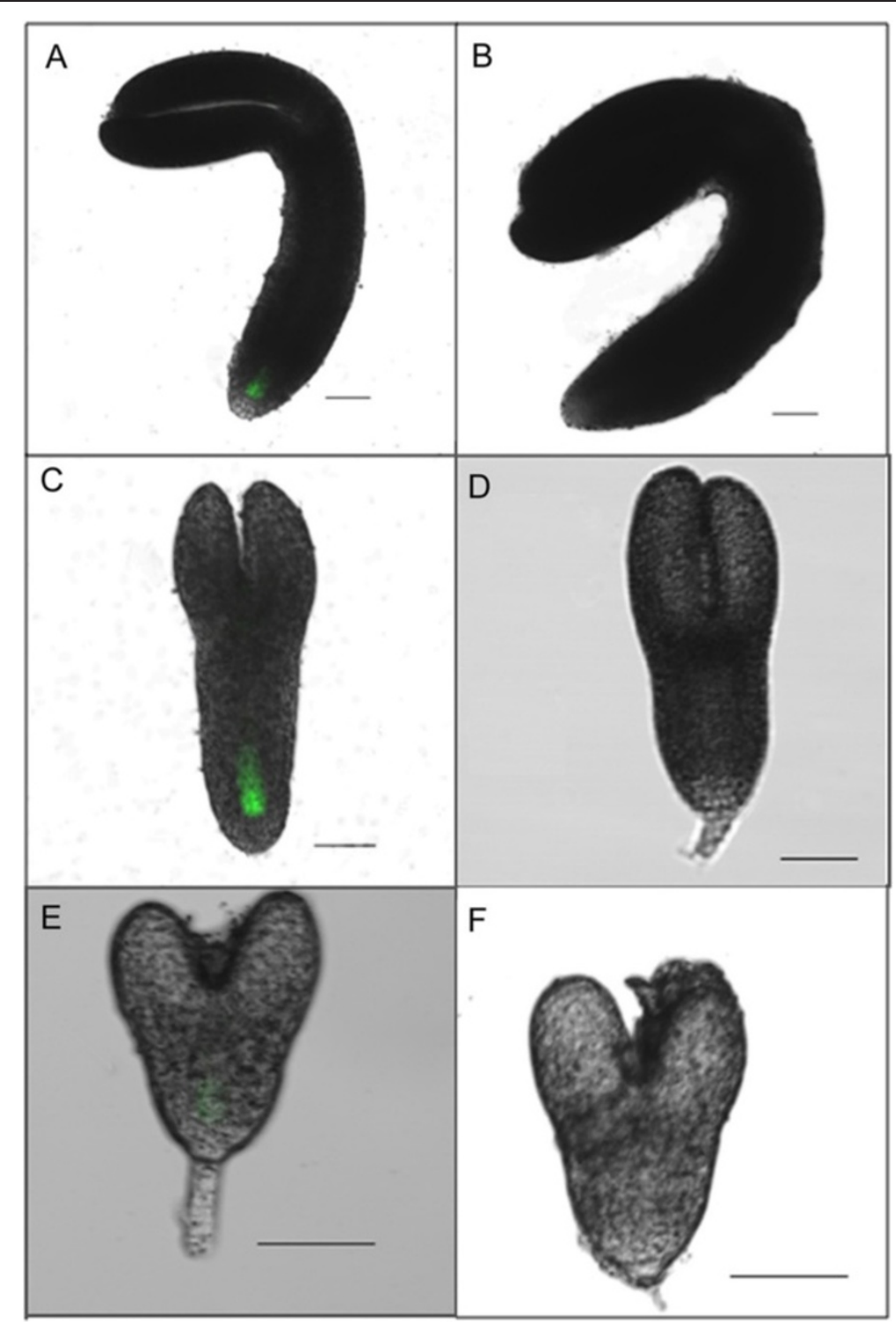

Figure 2 Identification of At4g32460 promoter activity during Arabidopsis thaliana embryo development using pBDX::ER-GFP plants (Additional file 1: Figure S1C). A) GFP fluorescence in provascular cells from radicle meristematic region of mature embryo. C) GFP fluorescence in provascular cells from radicle meristematic region of torpedo-stage embryo. E) GFP fluorescence in provascular cells from radicle meristematic region of heart stage embryo. B), D), and F) Images of wt embryo stages. Scale bars $=50 \mu \mathrm{m}$. All images are projections of confocal Z-stacks.

reported previously [13]. The same pattern of PME activity was observed in OE11420 seeds. At $20 \mathrm{~h}$ of germination, PME activity was significantly higher in OE11420 seeds than in wt seeds. However, the PME activity pattern for OEBDX seeds was different; the PME activity did not decrease at $34 \mathrm{~h}$ of germination. In the $1-\mathrm{h}$ and $34-\mathrm{h}$ germinating seeds, PME activity was higher in OEBDX seeds than in wt seeds $(p<0.001$, Figure $5 C)$.

Pectins are the main component of $A$. thaliana seed mucilage, and can be detected by staining with ruthenium red [28]. In water-imbibed OEBDX seeds, mucilage release was similar to that of wt imbibed seeds (Additional file 1: Figure S5).
Next, we used antisense RNA technology to silence $B D X$ expression. An At4g32460 RNA antisense transgene driven by the cognate promoter of the endogenous $B D X$ gene (BDX::BDX antisense RNA transgene; Additional file 1: Figure S1D) was transformed into wild-type $A$. thaliana plants to generate ASBDX plants (Figure 6). Five independent transgenic lines were obtained by kanamycin selection. All of these transgenic lines exhibited phenotypic variation in the $\mathrm{T} 1$ progeny, and the segregation analysis revealed a 3:1 ratio of wt phenotypes to defective phenotypes. This ratio was previously observed for an antisense construct with a cognate promoter in rice [29]. Analyses of morphological and developmental defects were conducted 
A

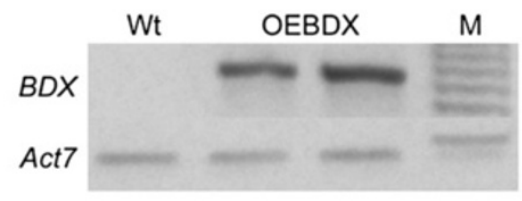

C

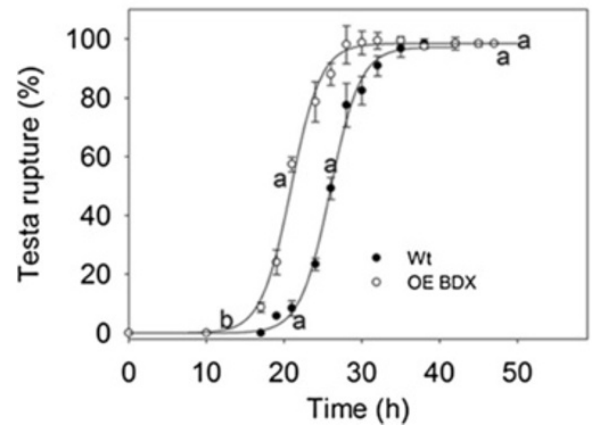

B

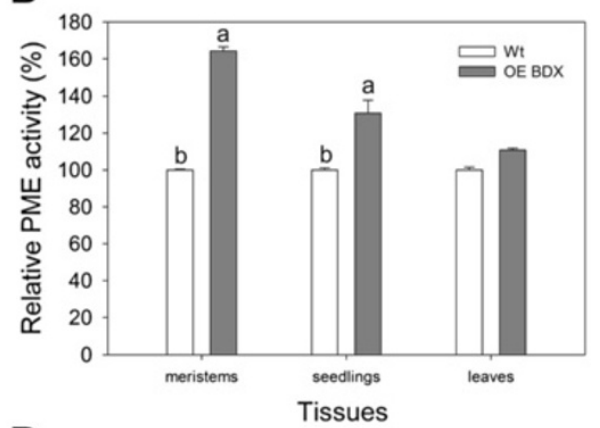

D

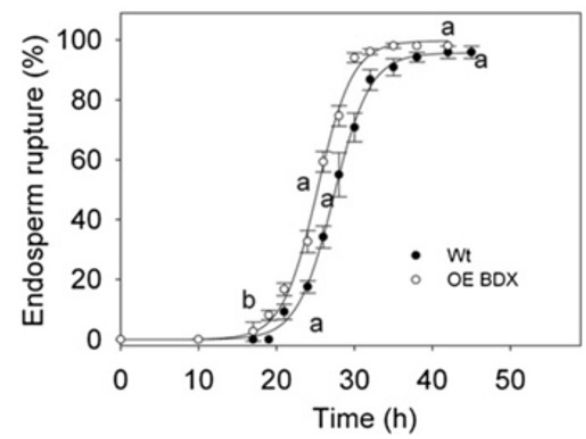

Figure 3 Effects of BDX overexpression on BDX transcript level, PME activity, and germination of matrix-primed seeds. A) Detection of $B D X$ transcripts in dry seeds from wt plants and two independent OEBDX lines. ACT7 expression was used as an internal control. B) PME activities in meristem and leaves of $A$. thaliana plants (18 days after sowing) and seedlings ( 5 days post-germination). C) Cumulative testa rupture curve. D) Cumulative endosperm rupture curve. Germination assays were performed in triplicate at $20^{\circ} \mathrm{C}$. All experiments were performed at least three times for each line, with different seed lots. Values shown are mean \pm standard error; different letters indicate significant differences among lines.

using three independent antisense lines. All three lines showed reduced silique size and did not produce seeds (Figure 6A, B, C and D). Analysis of a transverse section of a stage 13 ASBDX flower bud revealed abnormal carpel morphology. The size of the stigma was decreased, and the septum had engrossed regions. In mature ovules, different tissues could not be differentiated (Figure 6D, close-up image). The mean silique length for ASBDX lines 1, 2, and 3 , was $3.3,3.1$, and $4 \mathrm{~mm}$, respectively, corresponding to a size reduction of at least $60 \%$ (Figure $6 \mathrm{E}$ ). Compared with wt, all three ASBDX lines showed significantly lower PME activity in the leaves $(p=0.014, p=0.001$, and $p=0.003$ for lines 1, 2, and 3, respectively; Figure 6F). A quantitative reverse-transcription polymerase chain reaction (qRT-PCR) analysis revealed that all three ASBDX lines showed a $40 \%$ reduction in At4g32460 transcript levels, compared with that in wt inflorescences $(p<0.001$ for line 1 and 2, and $p=0.004$ for line 3; Figure 6G).

We conducted a phenotypic analysis of two SALK lines, SALK_142260 and SALK_054867, each of which have a T-DNA insertion in the $B D X$ coding sequence. The results provided further evidence that $B D X$ plays roles in reproductive development, and possibly in modulating PME (Figure 7). The T-DNA insertion in the
SALK_142260 line is at end of the second exon and that in the SALK_054867 line at the end of the third exon of the At4g32460 locus (Figure 7A). Heterozygous plants of the two SALK lines showed a reduction in silique length, similar to that in ASBDX plants (Figure $7 \mathrm{~B}$ and $\mathrm{D}$ ). However, in the SALK lines, the siliques contained a few seeds (average, $4 \pm 1$ seeds). Some seeds showed an abnormal morphology and were not viable (Figure 7C). Seeds with normal morphology generated either heterozygous or wt plants but not homozygous plants. A qRT-PCR analysis showed a significant decrease in $B D X$ transcript levels in T-DNA heterozygous plants $(p=003$, Figure 7E).

\section{Discussion}

DUF642 is a highly conserved family of cell wall-related proteins specific to spermatophytes. This family shows a high level of amino acid identity among different plant species, suggesting that members of the DUF642 play an important function in plant cell wall properties [1]. Our results showed that $B D X$ was expressed in embryos, in imbibed seeds, in 48-h seedlings, and throughout the adult plant, where it was primarily localized in vascular tissue, as has been described for At5g25460 [2] (Figures 1 and 2). $B D X$ was detected in the initial cells of the different stages 

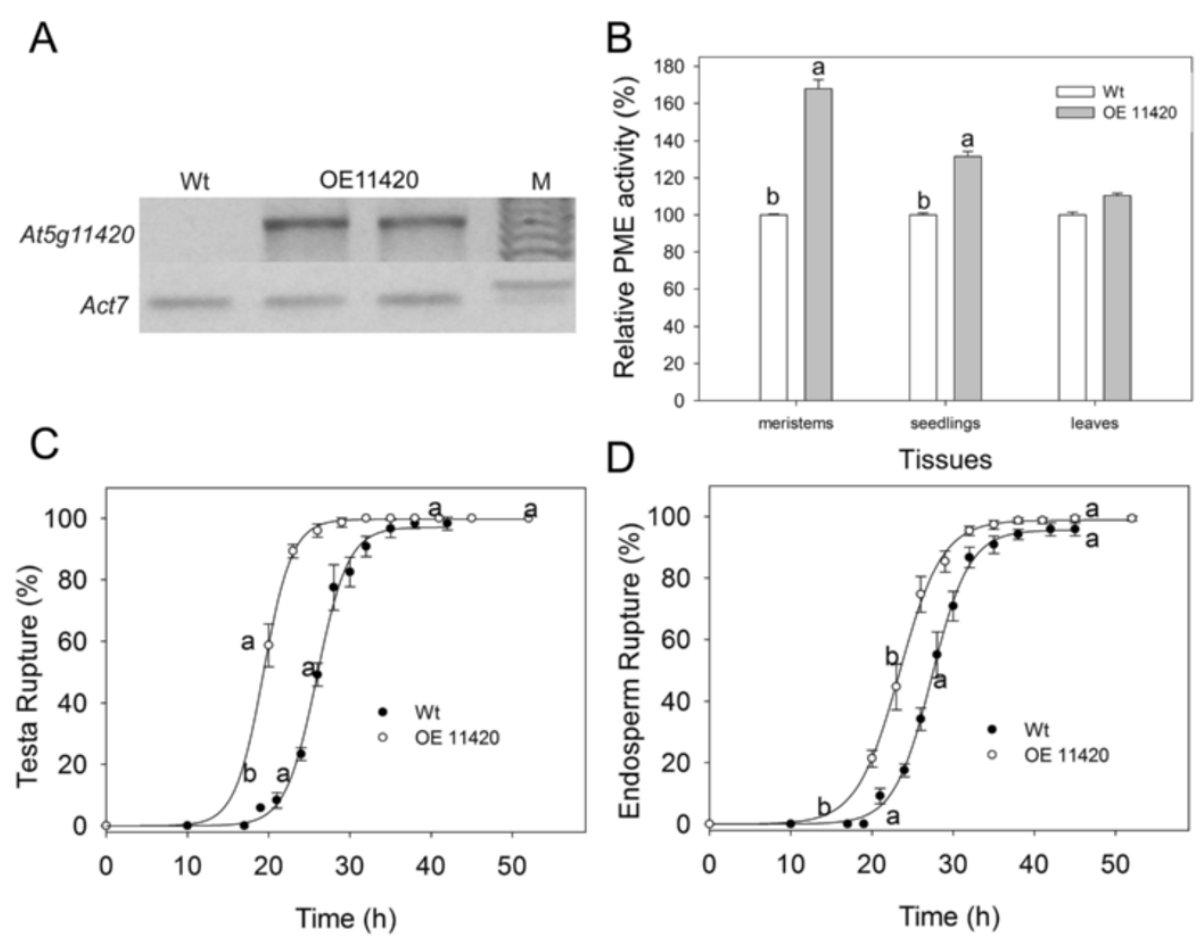

Figure 4 Effects of At5g11420 overexpression on At5g11420 transcript level, PME activity, and germination of matrix-primed seeds. A) At5g11420 transcript level in dry seeds of wt and two independent OE11420 lines. ACT7 expression served as the internal control. B) PME activities in meristem and leaves of $A$. thaliana plants (18 days after sowing) and seedlings ( 5 days post-germination). Values are means \pm standard error. C) Cumulative testa rupture curve. D) Cumulative endosperm rupture curve. Germination assays were performed in triplicate at $20^{\circ} \mathrm{C}$. Different letters indicate significant differences among lines. All experiments were performed at least three times for each line, with different seed lots.

of the embryo and primary root, mainly in the pericycle cells before lateral root emergence; therefore, its expression in vascular tissue in other organs could be related to these types of cells.

Auxin signaling promotes the emergence of lateral root primordia [30]. In the present study, auxin induced $B D X$ expression in roots (Additional file 1: Figure S2), and $B D X$ was highly expressed in stigma tissue of stage 12 flowers (Figure $1 \mathrm{H}$ ), where auxin-signaling genes are overrepresented [31]. Auxin distribution in the stigma plays an important role in the pollination process. In the ASBDX plants, the siliques were very short and contained no seeds; in the heterozygous T-DNA plants, the siliques were short but they contained a few seeds (Figures 6 and 7). The high transcript level of $B D X$ in stigma tissue could be related to the low seed yield of ASBDX and T-DNA plants. It has been suggested that the regulation of cell wall structure, and especially pectin status, in female tissue is fundamental for pollen tube penetration [32]. Previous studies have shown that methylesterification of the transmitting tract decreases before and during pollination and that PME activity increases during silique development $[17,18,32]$. Whereas pme3-null mutants showed no morphological changes in silique development [33], PMEI5 OE plants grew short and wrinkled siliques containing only one or two seeds. A decrease in total PME activity is detected in PMEI5 OE [13] and in ASBDX plants (Figure 6).

BDX was also expressed during embryogenesis (Figure 2). In three generations, homozygous T-DNA plants were not obtained, suggesting that there was an embryo development defect in null mutant plants.

Transgenic plants overexpressing AtPME3 showed an increase in total PME activity, but their only morphological changes are longer roots and taller shoots than those of wt [33]. In the present study, the $A$. thaliana plants overexpressing BDX and At5g11420 had no morphological changes, as compared with wt, during different vegetative stages of the life cycle, although they showed higher total PME activity in the meristem, seedlings, and imbibed seeds (Figures 3 and 4). The increased PME activity could be related to improved germination performance in OEBDX and OE11420 seeds. Compared with wt seeds, OEBDX and OE11420 seeds showed shorter initial times to testa rupture, which were correlated with the increase in PME activity (Figure 5). A previous study showed that the At5g11420 transcript is enriched in the micropylar endosperm before testa rupture [4]. In the present study, there were high levels of PME activity in the hours before testa rupture in the OE11420 line, 
A

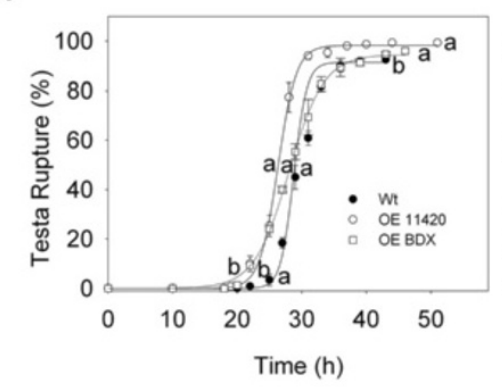

C

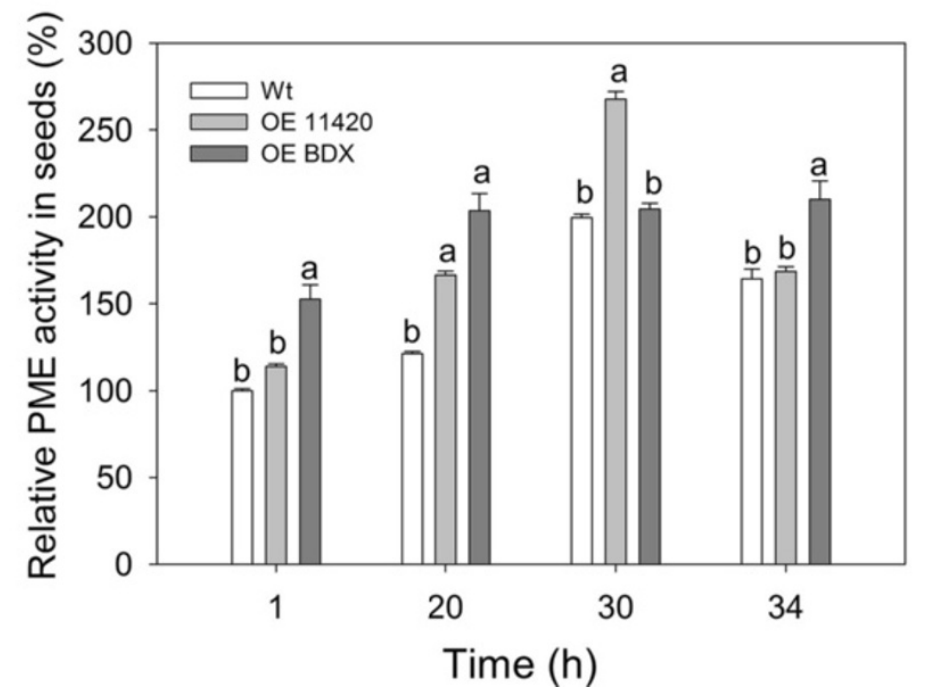

Figure 5 PME activity during germination of OEBDX and OE11420 control (dry) seeds. A) Cumulative testa rupture curve. B) Cumulative endosperm rupture curve. C) PME activity. PME activity assays were performed in triplicate. All experiments were performed at least twice for each line, with different seed lots. For each time point, letters indicate significant differences. Error bars show SE.

suggesting that At5g11420 plays a physiological role in germination.

\section{Conclusions}

Our data indicated that the DUF642 proteins encoded by $B D X$ and At5g11420 are positive regulators of PME activity in A. thaliana. They may affect PME activity directly, or indirectly via altering cell wall properties. The relevance of cell wall modifications during plant development has been studied extensively, and PMEs have been shown to play important roles in modulating methylesterification during fruit development and germination [34]. In this study, the lines overexpressing BDX and At5g11420 showed increased PME activity during seed germination, a two-step process that is highly correlated with changes in PME activity. We speculate that PME activation leads to faster completion of germination by improving the capacity for testa rupture. Previous studies have shown that reduced cell wall pectin methylesterification allows improved access of PG to degrade pectin and promote cell separation in the testa. In addition, the modulation of PG activity by PGIP was shown to inhibit germination [23].
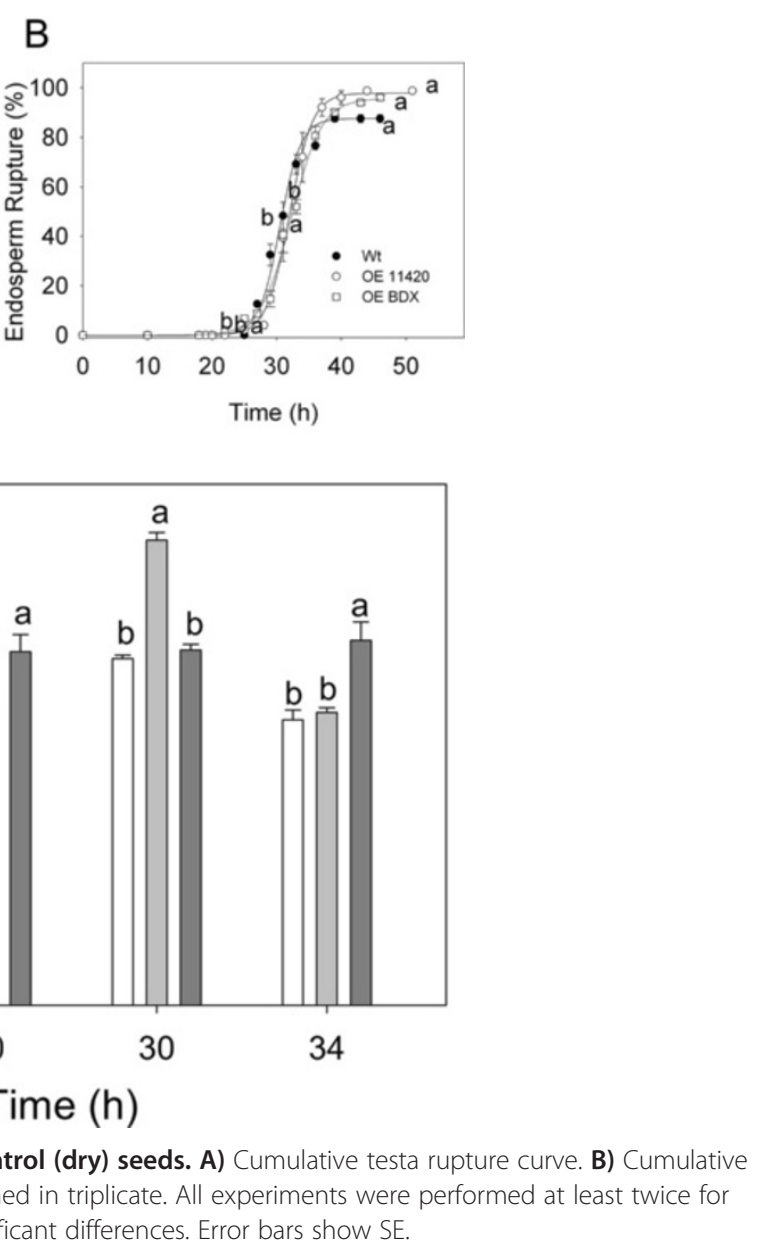

Many morphogenetic events during algal and plant development are related to pectin chemistry $[34,35]$. In Chara corallina, pectin de-esterification promotes cell expansion [36]. Phyllotaxis and organ initiation depend on the regulation of pectin status of the meristem [37]. During pollen tube elongation, a spatial gradient in pectin methyl-esterification is shown to be precisely controlled by PMEs and PMEI proteins [38].

The evolution of the complexity of the methylesterification process is an important process in plant diversification and adaptation to different environments. The expression of pectin and PME in cell walls first occurred in charophytes. The inhibitory domain of PMEs appeared in the PMEI family in land plants during the divergence of mosses from charophytes [39]. The cellulose binding protein (CBP) of the nematode Heterodera schachtii increases PME activity in plants through directly interacting with AtPME3; other than this exception, no other positive regulator of PMEs has been described [33]. Our results suggest that DUF642 proteins, a spermatophyte-specific family, contribute to the complexity of the methylesterification process by participating 


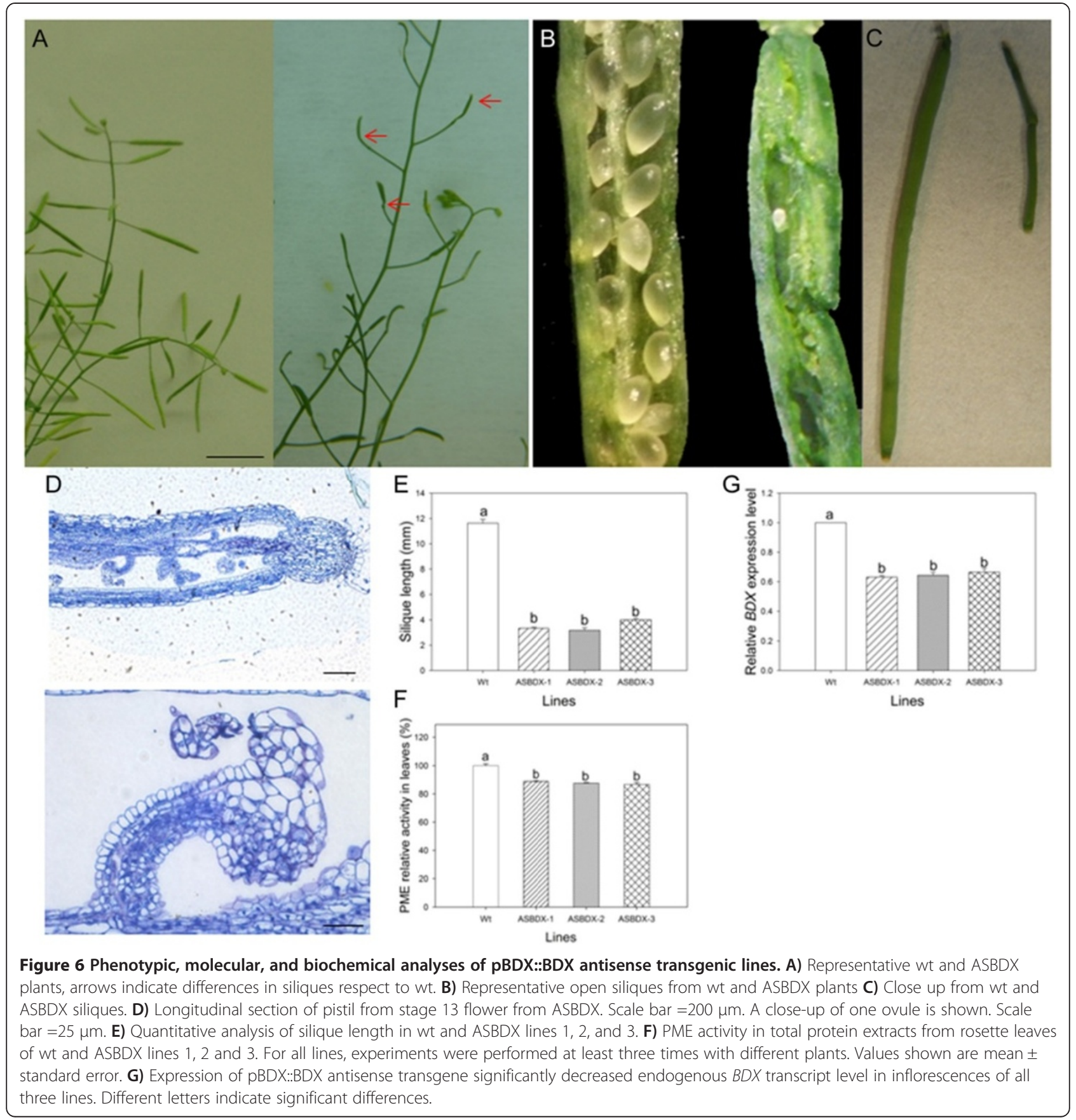

in the fine regulation of pectin status during plant development.

\section{Methods}

\section{Plant materials}

Wild-type Columbia (Col) ecotype A. thaliana plants (wt) and lines overexpressing (OE) At4g32460 and At5g1120 were sown in soil in pots, or on Murashige and Skoog (MS) medium ( $\mathrm{pH}$ 5.7) in Petri dishes. The plants were grown in a chamber (E-15, Conviron, Manitoba, Canada) at $21^{\circ} \mathrm{C}$ under a long-day photoperiod (16-h light/8-h dark). Five-day-old seedlings and vegetative meristems (comprising the young organ primordia and the shoot apical meristem proper) and rosette leaves (from 18day-old plants) were collected, frozen in liquid nitrogen, and stored at $-80^{\circ} \mathrm{C}$ until use in PME activity and RNA analyses.

To determine the expression patterns of At4g32460 and At5g11420 during imbibition, $50 \mathrm{mg}$ wt A. thaliana seeds were sown on $1 \%(\mathrm{w} / \mathrm{v})$ agar plates and placed in a 


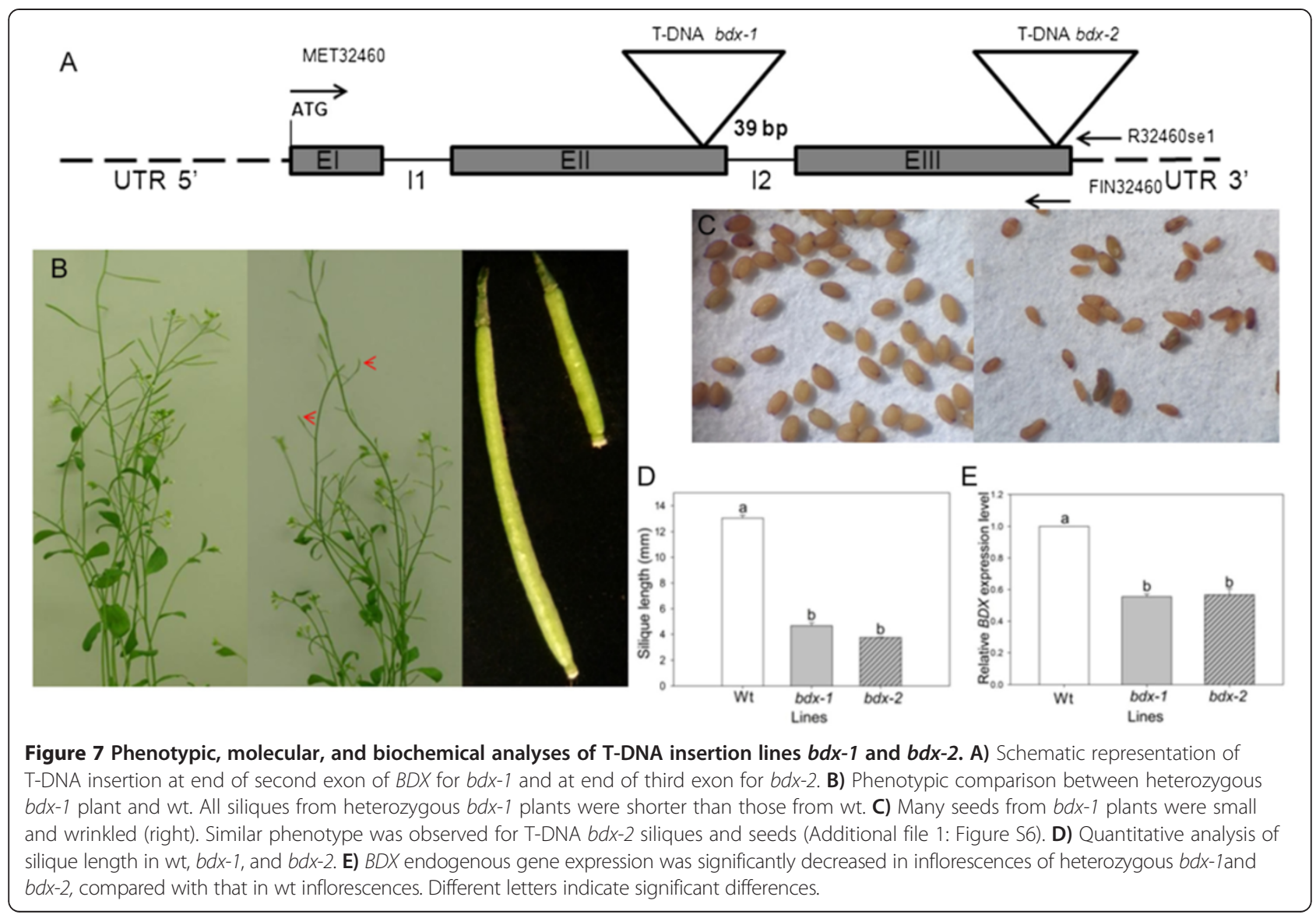

growth chamber (Lab-Line Instruments Inc. Melrose Park, IL, USA) at $21^{\circ} \mathrm{C}$ under a 16 -h light/8-h dark photoperiod. Seeds were collected at 2, 4, 6, 8, 12, 24, and $48 \mathrm{~h}$ post-imbibition, frozen in liquid nitrogen, and then stored at $-80^{\circ} \mathrm{C}$ until RNA extraction.

DNA extraction, RNA extraction, and seed cDNA synthesis Genomic DNA was extracted from rosette leaves and inflorescences using the phenol chloroform isoamyl alcohol method (Invitrogen, Carlsbad, CA, USA) according to the manufacturer's instructions. Total RNA was extracted from rosette leaves and inflorescences using the Trizol method (Invitrogen) according to the manufacturer's instructions.

Total RNA was isolated from $0.05 \mathrm{~g}$ A. thaliana wt and OE seeds according to the protocol described elsewhere [40]. A Nanodrop spectrophotometer (Nanodrop Lite; Thermo Scientific; www.thermoscientific.com) was used to quantify RNA. For semiquantitative RT-PCR, cDNA was synthesized from 100 ng RNA that had been treated with DNase-I (Qiagen, Valencia, CA, USA) using SuperScript II Reverse Transcriptase (Invitrogen) and oligo (dT) primers. We used the primer pair FAT32460 (forward, 5'-GTGTCCCAAAGCCATTATTC-3') and RAT 32460 (reverse, 5' -AGCGACGAATCTCAATGAC-3') to amplify At4g32460, and the primer pair 11420LSF (forward, 5' -TCTAGAATGAAAGGAGGCAGCCTCT-3') and 1142 OLSR2 (reverse, 5'-GGATCCCGGCTTACGAGCACTGA GGAGTT-3') to amplify At5g11420. Actin (ACT7) was used as an internal control.

\section{Quantitative RT-PCR}

cDNA samples synthesized from 100 ng RNA from antisense, T-DNA, and wt inflorescences were used for amplification with SYBR Green Master Mix using an Applied Biosystems StepOne platform (Applied Biosystems, Foster City, CA, USA). The PCR conditions were as follows: $50^{\circ} \mathrm{C}$ for $2 \mathrm{~min}$ for DNA polymerase activation, $95^{\circ} \mathrm{C}$ for $10 \mathrm{~min}$, followed by 40 cycles of $15 \mathrm{~s}$ at $95^{\circ} \mathrm{C}$ and $1 \mathrm{~min}$ at $60^{\circ} \mathrm{C}$; finally, samples were subjected to $15 \mathrm{~s}$ at $95^{\circ} \mathrm{C}, 1 \mathrm{~min}$ at $60^{\circ} \mathrm{C}$, and $15 \mathrm{~s}$ at $95^{\circ} \mathrm{C}$ for melting curve analysis. Three independent biological replicates with three technical replicates were analyzed. ACT7 served as the endogenous control. To analyze gene transcript levels in inflorescences of antisense plants, the forward primer consisted of a sequence from the UTR $5^{\prime}$ region $\left(5^{\prime}\right.$-CTCTCGCTC ACTCTTCTCCAA-3') and the reverse primer consisted of a sequence from the beginning of the second exon (5'-CGACAAAGCCTGAGAGTTCCC-3', BDXR). For analyses of the T-DNA lines, we used the $B D X$ forward 
primer (5'-GCTTCAATGATGGACTACTACC-3', BDXF) and BDXR. Samples were compared with $\mathrm{C}_{\mathrm{T}}$ and slope values and analyzed using the mathematical model established by Pfaffl [41] to obtain the relative expression ratio. Data were subjected to a natural log transformation before the Student's $t$-test.

\section{Plasmid construction}

For At4g32460 (GENE ID 829381) expression analysis, a 1983-bp fragment of the At4g32460 intergenic region ending at ATG was amplified from leaf-extracted genomic DNA using the following primers: PRO32460F (forward, 5'-AAGCTTGCATGGGAGAATTGACCACT-3') and PRO32460R (reverse, 5'-GGATCCTTGGAGAAGA GTGAGCGAGAG-3'). This PCR fragment was cloned into pGEM-T Easy and then sequenced. The fragment was then partially digested with HindIII followed by BamHI, and then cloned into the pBIN-m-GFP-ER plasmid (PRO32460: ER-GFP, Figure 1).

For overexpression of At4g32460 and At5g11420 (GENE ID 831013), their coding regions were amplified from cDNA synthesized from leaf mRNA using the primers pairs F32460se1 (forward, 5'-GGATCCATGAA AGAGATGGGAGTGATAG-3') and R32460se1 (reverse, 5'-GAGCTCTCACGGCCTCCGAGCACT-3'); and F1 1420se1 (forward, 5'-GGATCCATGAAAGGAGGCA GCCTCT-3') and R11420se1 (reverse, 5'-GAGCTCT TACGGCTTACGAGCACTGA-3'), respectively. These PCR fragments were cloned into pGEM-T Easy and then sequenced. After digestion with BamHI and SacI, the fragments were subcloned into the pBIN plasmid and expressed under the control of the CaMV 35S promoter (Figure $1 \mathrm{C}$ and $\mathrm{D}$ ). For gene silencing analysis, we constructed a plasmid with the At4g32460 antisense transgene under the control of its cognate promoter.

\section{Plant transformation}

Wt Col $A$. thaliana plants were transformed using the floral-dip method via Agrobacterium tumefaciens C58 [42]. Collected seeds were sown on plates containing MS medium ( $\mathrm{pH}$ 5.7) with kanamycin for selection, and green seedlings were transplanted into soil in pots. At least 10 independent transgenic lines were selected. The homozygous T3 seed generation was used for germination experiments (two independent lines) or confocal microscopy (three independent lines). For antisense transgenic plants, at least 10 independent lines were selected. For phenotype analysis, five homozygous (T3) lines were used, and three lines were used for PME activity and qRT-PCR analyses.

\section{T-DNA lines}

The insertion in the T-DNA SALK_142260 line was verified by PCR, and the region of interest was sequenced using the
T-DNA left border primer LBb1 (5'-GCGTGGACCGCTT GCTGCAACT-3') and a specific primer for $B D X$, R32460se1, located at the end of the second exon. For the SALK_054867 line, the reverse primer consisted of a sequence located at the end of the third exon.

\section{Laser confocal scanning microscopy}

To label the cell walls, seedlings were incubated in a propidium iodide solution $(1.7 \mathrm{mM})$ for $30 \mathrm{~s}$ before confocal imaging analysis. Propidium iodide and GFP were imaged at 485 and $545 \mathrm{~nm}$, respectively, using an aFV100 Laser Confocal Scanning Microscope (Olympus, Tokyo, Japan). Images from all plant tissues without fluorescence were used as controls. The images were assembled using Photoshop, v. 5.0 (Adobe Systems, San Jose, CA, USA).

\section{Matrix priming treatment (M)}

A. thaliana seeds were enclosed in cellophane and buried at $2 \mathrm{~cm}$ depth in a pot filled with field-capacity humid soil (-0.027 MPa water potential). The pot was covered with aluminum foil and incubated at $22^{\circ} \mathrm{C}$ for $24 \mathrm{~h}$. Seeds were exhumed in the dark and air-dried at room temperature $\left(25^{\circ} \mathrm{C}\right)$. Subsequently, the seeds were used for germination testing or frozen in liquid nitrogen and stored at $-80^{\circ} \mathrm{C}$.

\section{Germination testing}

For germination testing, dry mature seeds were stored at $20^{\circ} \mathrm{C}$ for 3 months of after-ripening. Seeds were sown on $1 \%(\mathrm{w} / \mathrm{v})$ agar plates and transferred to a plant growth chamber (Lab-Line Instruments Inc.) at $20^{\circ} \mathrm{C}$ under a 6 -h light/18-h dark photoperiod. All germination experiments consisted of five replicates with 30 seeds per replicate. Biological replicates were performed with different seed lots of wt and the different overexpression lines for $B D X$ and At5g11420. Germination was scored using an Olympus dissection microscope. Seeds with visible endosperm were considered to have reached testa rupture. Seeds with a radicle tip emerging through the endosperm were considered to have reached endosperm rupture. The percentages of seeds with testa and endosperm rupture over time were fitted to sigmoid models using Table Curve 2D v.3 software (AISN, Software, Chicago, IL, USA). From these models, we obtained the lag time of germination and the germination rate after an arcsine transformation to meet the assumptions of the test. The results were analyzed with an analysis of variance (ANOVA) and Tukey's post hoc test (Sigma Plot v.11, Systat Software Inc., San José, CA, USA). Germination percentages were also arcsine-transformed to meet the assumptions of the test. To compare the slope between wt and OE lines, the testa and endosperm fits were included. 


\section{PME activity assays}

PME activity was determined according to a previously described method [28] with the following modifications: 8-20 $\mu \mathrm{g}$ protein in equal volumes $(5-20 \mu \mathrm{l})$ was loaded into a gel matrix prepared in $50-\mathrm{mm}$ Petri dishes (instead of 6-mm-diameter wells). The gel consisted of $0.1 \%(\mathrm{w} / \mathrm{v}) \geq 85 \%$ esterified citrus fruit pectin (Sigma-Aldrich), 1\% (w/v) agarose, $12.5 \mathrm{mM}$ citric acid, and $50 \mathrm{mM} \mathrm{Na}_{2} \mathrm{HPO}_{4}(\mathrm{pH}$ 6.5). The PME activities in different tissues were normalized to the corresponding wild-type average activity (=100). Significant differences were assessed using a Mann-Whitney $U$ test and two-way multivariate analysis of variance (MANOVA) for seed imbibition.

\section{Histological analyses}

Silique samples from plants harboring the antisense construct were fixed in FAA (by volume, $4 \%$ formaldehyde, $2 \%$ acetic acid, $50 \%$ ethanol). The samples were dehydrated through a graded ethanol series and embedded in paraffin. Sections $(1-2 \mu \mathrm{m})$ were cut with a rotary microtome, and then stained with toluidine blue. Histological sections were observed and photographed under an Olympus microscope.

\section{Availability of supporting data}

The supporting data of this article is included in the additional file.

\section{Additional file}

Additional file 1: Figure S1. Schematic presentation of transgene constructs. (A, B) At4g32460 and At5g11420 CDNA fragments were cloned into pBIN to generate CAMV 35S::BDX (OEBDX) and CAMV 35S::At5g 11420 (OE11420) constructs, respectively. (C) To construct pBDX::mGFP-ER, a 1983-bp fragment of At4g32460 intergenic region was cloned into pBIN-m-GFP-ER. (D) pBDX::BDX antisense consisted of At4g32460 RNA antisense transgene driven by the cognate promoter of At4g32460 (ASBDX). Black arrow, CaMV promoter; striped arrows, cognate promoters: gray arrows (right), sense fragments; gray arrows (left), antisense fragment. Figure S2. Early and differential transcription of BDX and At5g11420 during seed imbibition. ACT7 was analyzed as internal standard. Figure S3. Auxin- and gibberellic acid-inducible expression of At4g32460 in primary roots. Transgenic 5-day-old seedlings were treated for $0 \mathrm{~h}(\mathbf{A}, \mathbf{D})$ or $24 \mathrm{~h}$ with $2 \mu \mathrm{M} \mathrm{GA}(\mathrm{B}, \mathrm{E})$ or for $48 \mathrm{~h}$ with $2 \mu \mathrm{M}$ IAA (C,F). A) GFP fluorescence in provascular tissue of meristematic zone (as in Figure 2). B) GFP fluorescence in provascular and vascular tissue of meristematic and transition zone. C) GFP fluorescence in provascular and vascular tissue of meristematic and transition zone and was also detected in cortical cells of transition zone. D) GFP fluorescence in pericycle cells of vascular tissue of maturation zone (as in Figure 2). E) GFP fluorescence in vascular tissue and cortical cells of maturation zone. F) GFP fluorescence in vascular tissue and cortical cells of maturation zone. Figure S4. Effect of empty plasmid on germination performance. A) Cumulative testa rupture curve. B) Cumulative endosperm rupture curve. Figure S5. OEBDX and OE11420 seeds did not show defective mucilage release after imbibition in water [43]. Figure S6. Phenotypic analyses of $b d x-2$. A) Phenotypic comparison between heterozygous $b d x-2$ plant and Siliques of heterozygous $b d x-2$ plants were shorter than those of wt. B) Many seeds from $b d x-2$ plants were small and wrinkled (right).

\section{Abbreviations}

BDX: BIIDXI; DUF642: Domain unknown function 642; PME: Pectin Methyl Esterase; AtPME3: Pectin Methyl Esterase 3 from Arabidopsis thaliana; PMEl: PME inhibitor; HGs: Homogalacturanans; PG: Polygalacturonase; PGIP: Polygalacturonase inhibitors proteins; ABA: Abscisic acid; GFP: Green fluorescent protein; GA: Gibberelic acid; GARE: Gibberelic acid response elements; OE: Overexpression; OEBDX: BDX Overexpression; ASBDX: Antisense BDX; CBP: Cellulose Binding Protein; Col: Columbia ecotype; wt: Wild-type; MS: Murashigee and Skoog; IAA: Indole-3-acetic acid.

\section{Competing interests}

The authors declare that they have no competing interests.

\section{Authors' contributions}

EZ and DS carried out the molecular genetic studies and participated in germination analysis. $\mathrm{EMB}$ and $\mathrm{AOS}$ participated in the design of the study. AGB conceived of the study and participated in its design and coordination $E Z, D S, E M B, A O S$ and $A G B$ wrote the article. All authors read and approved the final manuscript.

\section{Acknowledgments}

This paper constitutes a partial fulfillment of the Posgrado en Ciencias Biomédicas, Universidad Nacional Autónoma de México (UNAM). E. Zúñiga-Sánchez acknowledges the scholarship and financial support provided by the Consejo Nacional de Ciencia y Tecnología (CONACyT) and DGAPA-UNAM.

This work was supported by the Programa de Apoyo a Proyectos de Investigación e Innovación Tecnológica (PAPIIT) IN200912 (Universidad Nacional Autónoma de México) and SEP CONACyT grant 155074. D. Soriano has received a postdoctoral fellowship from CONACyT (3910). We thank G. Cassab for discussion during the course of this work and $K$ Jiménez for assistance on Confocal studies, L. Márquez for DNA sequencing, M.K. Pérez Pacheco for histological studies, R. Pérez Ruíz for his advice on QPCR analysis and M.M. Garciadiego San Juan for PME activity analysis. No conflicts of interest declared.

\section{Author details}

${ }^{1}$ Instituto de Ecología, Universidad Nacional Autónoma de México, Apartado Postal 70-275, Ciudad Universitaria, México 04510, Distrito Federal Mexico. 2Facultad de Química, Universidad Nacional Autónoma de México, Ciudad Universitaria, México 04510, Distrito Federal Mexico.

Received: 25 June 2014 Accepted: 17 November 2014

Published online: 02 December 2014

\section{References}

1. Vázquez-Lobo A, Roujol D, Zúñiga-Sánchez E, Albenne C, Pinero D, Gamboa-deBuen A, Jamet E: The highly conserved spermatophyte cell wall DUF642 protein family: phylogeny and first evidence of interaction with cell wall polysaccharides in vitro. Mol Phylogenet Evol 2012, 63:510-520.

2. Gao Y, Badejo AA, Sawa Y, Ishikawa T: Analysis of two L-galactono-1,4-lactone responsive genes with complementary expression during the development of Arabidopsis thaliana. Plant Cell Physiol 2012, 53:592-601.

3. Sreenivasulu N, Usadel B, Winter A, Radchuk V, Scholz U, Stein N, Weschke W, Strickert M, Close TJ, Stitt M, Graner A, Wobus U: Barley grain maturation and germination: metabolic pathway and regulatory network commonalities and differences highlighted by new MapMan/PageMan profiling tools. Plant Physiol 2008, 146:1738-1758.

4. Dekkers BJ, Pearce S, van Bolderen-Veldkamp RP, Marshall A, Widera P, Gilbert J, Drost HG, Bassel GW, Müller K, King JR, Wood AT, Grosse I, Quint M, Krasnogor N, Leubner-Metzger G, Holdsworth MJ, Bentsink L: Transcriptional dynamics of two seed compartments with opposing roles in Arabidopsis seed germination. Plant Physiol 2013, 163:205-215.

5. Soeda Y, Konings MC, Vorst O, van Houwelingen AM, Stoopen GM, Maliepaard CA, Kodde J, Bino RJ, Groot SP, van der Geest AH: Gene expression programs during Brassica oleracea seed maturation, osmopriming, and germination are indicators of progression of the germination process and the stress tolerance level. Plant Physiol 2005, 137:354-368.

6. Garza-Caligaris LE, Avendaño-Vázquez AO, Alvarado-López S, Zúñiga-Sánchez E, Orozco-Segovia A, Pérez-Ruiz RV, Gamboa-deBuen A: At3g08030 transcript: a molecular marker of seed ageing. Ann Bot 2012, 110:1253-1260. 
7. Jamet E, Canut H, Bougart G, Pont Lezica RF: Cell wall proteins: a new insight through proteomics. Trends Plant Sci 2006, 11:33-39.

8. Tung CW, Dwyer KG, Nasrallah ME, Nasrallah JB: Genome-wide identification of genes expressed in Arabidopsis pistils specifically along the path of pollen tube growth. Plant Physiol 2005, 138:977-989.

9. Rejón JD, Delalande F, Schaeffer-Reiss C, Carapito C, Zienkiewicz K, de Dios AJ, Rodríguez-García MI, Van Dorsselaer A, Castro AJ: Proteomics profiling reveals novel proteins and functions of the plant stigma exudate. J Exp Bot 2013, 64:5695-5705.

10. Zúñiga-Sánchez E, Gamboa-deBuen A: The two DUF642 At5g11420 and At4g32460- encoded proteins interact in vitro with the AtPME3 catalytic domain. In Protein Interaction/Book 1. Edited by Cai J, Wang RE. Rijeka: InTech Editorial; 2012. ISBN 979-953-307-5777.

11. Mewalal R, Mizrachi E, Mansfield SD, Myburg AA: Cell wall-related proteins of unknown function: missing links in plant cell wall development. Plant Cell Physiol 2014, 6:1031-1043

12. Peaucelle A, Braybrook SA, Le Guillou L, Bron E, Kuhlemeier C, Höfte H: Pectin induced changes in cell wall mechanics underlie organ initiation in Arabidopsis. Curr Biol 2011, 21:1720-1726.

13. Müller K, Levesque-Tremblay G, Bartels S, Weitbrecht K, Wormit A, Usadel B, Haughn G, Kermode AR: Demethylesterification of cell wall pectins in Arabidopsis plays a role in seed germination. Plant Physiol 2013, 161:305-316.

14. Hongo S, Sato K, Yokoyama R, Nishitani K: Demethylesterification of the primary wall by PECTIN METHYLESTERASE35 provides mechanical support to the Arabidopsis stem. Plant Cell 2012, 24:2624-2634.

15. Di Matteo A, Giovane A, Raiola A, Camardella L, Bonivento D, De Lorenzo G, Cervone F, Bellincampi D, Tsernoglou D: Structural basis for the interaction between pectin methylesterase an a specific inhibitor protein. Plant Cell 2005, 17:849-858.

16. González-Carranza ZH, Elliott KA, Robert JA: Expression of polygalacturonases and evidence to support their role during cell separation processes in Arabidopsis thaliana. J Exp Bot 2007, 58:3719-3730.

17. Suárez C, Zienkiewicz A, Castro AJ, Zienkiewicz K, Majewska-Sawka A, Rodríguez-García MI: Cellular localization and levels of pectins and arabinogalactan proteins in olive (Olea europaea L.) pistil tissues during development: implications for pollen-pistil interaction. Planta 2013, 237:305-319.

18. Louvet R, Rayon C, Domon JM, Rusterucci C, Fournet F, Leaustic A, Crépeau MJ, Ralet MC, Rihouey C, Bardor M, Lerouge P, Gillet F, Pelloux J: Major changes in the cell wall during silique development in Arabidopsis thaliana. Phytochem 2011, 72:59-67

19. Lee KJ, Dekkers BJ, Steinbrecher T, Walshs $C T$, Bacic A, Bentsink L, Leubner-Metzger G, Knox JP: Distinct cell wall architectures in seed endosperms in representatives of the Brassicaceae and Solanaceae. Plant Physiol 2012, 160:1551-1566.

20. Nakabayashi K, Okamoto M, Koshiba T, Kamiya Y, Nambara E: Genome-wide profiling of stored mRNA in Arabidopsis thaliana seed germination: epigenetic and genetic regulation of transcription in seed. Plant J 2005, 41:697-709.

21. Ren $\mathrm{CH}$, Kermode AR: An increase in pectin methyl esterase activity accompanies dormancy breakage and germination of yellow cedar seeds. Plant Physiol 2000, 124:231-242.

22. Weitbrecht K, Müller K, Leubner-Metzger G: First off the mark: early seed germination. J Exp Bot 2011, 62:3289-3309.

23. Kanai M, Nishimura M, Hayashi M: A peroxisomal ABC transporter promotes seed germination by inducing pectin degradation under the control of ABI5. Plant J 2010, 62:936-947.

24. Goda H, Sawa S, Asami T, Fujioka S, Shimada Y, Yoshida S: Comprehensive comparison of auxin-regulated and brassinosteroid regulated genes in Arabidopsis. Plant Physiol 2004, 134:1555-1573.

25. Ogawa M, Hanada A, Yamauchi Y, Kuwahara A, Kamiya Y, Yamaguchi S: Gibberellin biosynthesis and response during Arabidopsis seed germination. Plant Cell 2003, 15:1591-1604.

26. Baumann K, De Paolis A, Costantino P, Gualberti G: The DNA binding site of the Dof protein NtBBF1 is essential for tissue-specific and auxinregulated expression of the rolB oncogene in plants. Plant Cell 1999, 11:323-334

27. Bastian R, Dawe A, Meier S, Ludidi N, Bajic VB, Gehring C: Gibberelic acid and CGMP-dependent transcriptional regulation in Arabidopsis thaliana. Plant Sign Beh 2010, 5:224-232.
28. Saez-Aguayo S, Ralet MC, Berger A, Botran L, Ropartz D, Marion-Poll A, North HM: PECTIN METHYLESTERASE INHIBITOR6 promotes Arabidopsis mucilage release by limiting methylesterification of homogalacturonan in seed coat epidermal cells. Plant Cell 2013, 25:308-323.

29. Li J, Jiang D, Zhou H, Li F, Yang J, Hong L, Fu X, Li Z, Liu Z, Li J, Zhuang C: Expression of RNA-interference/antisense transgenes by the cognate promoters of target genes is a better gene-silencing strategy to study gene functions in rice. PLoS One 2011, 6:e17444.

30. Overvoorde P, Fukaki H, Beeckman T: Auxin control of root development. In Cold Spring Harbor Perspectives in Biology: Auxin Signaling from Synthesis to Systems Biology. Edited by Estelle M, Weijers D, Ljung K, Leyser O. New York: Cold Spring Harbor Laboratory Press; 2011. ISBN 978-0-87969-898-0.

31. Sundberg E, Ostergaard L: Distinct and dynamic auxin activities during reproductive development. In Cold Spring Harbor Perspectives in Biology: Auxin Signaling from Synthesis to Systems Biology. Edited by Estelle M, Weijers D, Ljung K, Leyser O. New York: Cold Spring Harbor Laboratory Press; 2011. ISBN 978-0-87969-898-0.

32. Lehner A, Dardelle F, Soret-Morvan O, Lerouge P, Driouich A, Mollet JC: Pectins in the cell wall of Arabidopsis thaliana pollen tube and pistil. Plant Signal Behav 2010, 5:1282-1285.

33. Hewezi T, Howe P, Maier TR, Hussey RS, Mitchum MG, Davis EL, Baum TJ: Cellulose binding protein from the parasitic nematode Heterodera schachtii interacts with Arabidopsis pectin methylesterase: cooperative cell wall modification during parasitism. Plant Cell 2008, 20:3080-3093.

34. Wolf $\mathrm{S}$, Mouille G, Pelloux J: Homogalacturonan methyl esterification and plant development. Mol Plant 2009, 2:851-860.

35. Sénéchal $F$, Wattier $C$, Rustérucci $C$, Pelloux J: Homogalacturonan-modifying enzymes: structure, expression, and roles in plants. J Exp Bot 2014 65:5125-5160.

36. Proseus TE, Boyer JS: Calcium pectate chemistry controls growth rate of Chara corallina. J Exp Bot 2003, 57:3989-4002

37. Peaucelle A, Louvet R, Johansen JN, Höfte H, Laufs P, Pelloux J, Mouille G: Arabidopsis phyllotaxis is controlled by the methyl-esterification status of cell wall pectins. Curr Biol 2008, 18:1943-1948.

38. Hepler PK, Rounds CM, Winship LJ: Control of cell wall extensibility during pollen tube growth. Mol Plant 2013, 6:998-1017.

39. Wang M, Yuan D, Gao W, Li Y, Tan J, Zhang X: A comparative genome analysis of PME and PMEI families reveals the evolution of pectin metabolism in plant cell walls. PLoS One 2013, 8:e72082.

40. Oñate-Sánchez V, Vicente-Carbajosa J: DNA-free RNA isolation protocols for Arabidopsis thaliana, including seeds and siliques. BMC Res Notes 2008, 1:93.

41. Pfaffl MW: A new mathematical model for relative quantification in real-time RT-PCR. Nucleic Acid Res 2001, 29:e45.

42. Clough SJ, Bent AF: Floral dip: a simplified method for Agrobacterium-mediated transformation of Arabidopsis thaliana. Plant J 1998, 16:735-743.

43. Harpaz-Saad S, McFarlane HE, Xu S, Divi UK, Forward B, Western TL, Kieber JJ: Cellulose synthesis via the FEI2 RLK/SOS5 pathway and Cellulose synthase 5 is required for the structure of seed coat mucilage in Arabidopsis. Plant $J$ 2011, 68:941-953.

\section{doi:10.1186/s12870-014-0338-8}

Cite this article as: Zúñiga-Sánchez et al:: BIIDXI, the At4g32460 DUF642 gene, is involved in pectin methyl esterase regulation during Arabidopsis thaliana seed germination and plant development. BMC Plant Biology 2014 14:338. 